Title:

\title{
ACQUISITION, RETENTION AND TRANSFER OF SIMULATED LAPAROSCOPIC TASKS USING FNIR AND A CONTEXTUAL INTERFERENCE PARADIGM
}

\section{Author Names/Emails/Institutional Affiliation:}

\section{Name}

Patricia A. Shewokis, $\mathrm{PhD}^{1,2,3}$

Faiz U. Shariff, $\mathrm{MD}^{1}$

Yichuan Liu $^{2}$

Hasan Ayaz, $\mathrm{PhD}^{2,4,5}$

Andres Castellanos, $\mathrm{MD}^{1}$

D. Scott Lind, $M D^{1}$
Email

pas38@drexel.edu

fizashariff@gmail.com

yl565@drexel.edu

hasan.ayaz@drexel.edu

andres.castellanos@drexelmed.edu

david.lind@jax.ufl.edu

\section{Institutional Affiliations:}

${ }^{1}$ Department of Surgery, Drexel University College of Medicine. Philadelphia, PA 19102

${ }^{2}$ School of Biomedical Engineering, Science and Health Systems, Drexel University, Philadelphia, PA 19104

${ }^{3}$ College of Nursing and Health Professions, Drexel College of Medicine, Philadelphia, PA 19102

${ }^{4}$ Department of Family and Community Health, University of Pennsylvania, Philadelphia, PA, 19104

${ }^{5}$ Division of General Pediatrics, Children's Hospital of Philadelphia, Philadelphia, PA 19104

\section{Corresponding Author:}

Patricia A. Shewokis, PhD

Drexel University

3rd Floor, Room 382, Parkway Building

1601 Cherry Street

Mail Stop 31030

Philadelphia, PA 19102

Phone: 267-359-5827

FAX: 267-359-5843

Email: shewokis@drexel.edu

\section{Present address:}

D. Scott Lind, MD

Chair \& Professor, Department of Surgery

University of Florida College of Medicine-Jacksonville

3rd Floor, Faculty Clinic

53 West 8th Street, FC12

Jacksonville, FL 32209 


\section{Abstract}

BACKGROUND: Using functional near infrared spectroscopy, a noninvasive, optical brain imaging tool that monitors changes in hemodynamics within the prefrontal cortex (PFC), within a contextual interference paradigm, we assessed performance and cognitive effort during the acquisition, retention and transfer of multiple simulated laparoscopic tasks by novice learners.

METHODS: Third-year medical students $(n=10)$ were randomized to either a blocked or random practice schedule. Across 3 days, students performed 108 acquisition trials of 3 laparoscopic tasks on the LapSim $^{\circledast}$ simulator followed by delayed retention and transfer tests. .. Performance metrics (Global score, Total time) and hemodynamic responses (total hemoglobin $(\mu \mathrm{m})$ ) were assessed during skill acquisition; retention and transfer..

RESULTS: All acquisition tasks resulted in significant practice schedule $X$ trial block interactions for the left medial anterior PFC. During retention and transfer, random performed the skills in less time and had lower total hemoglobin change in the right dorsolateral PFC than blocked.

CONCLUSIONS: Compared with blocked, random practice resulted in enhanced learning through better performance and less cognitive load for retention and transfer of simulated laparoscopic tasks.

\section{SUMMARY SENTENCE}

We applied a contextual interference paradigm to assess performance and cognitive effort during the acquisition, retention and transfer of simulated laparoscopic tasks. Optical brain imaging complimented standard performance measures used to assess novice skill acquisition in a laparoscopic virtual reality simulator.

\section{KEYWORDS}

fNIR, Contextual Interference, Laparoscopic Skill Acquisition, Simulation. 


\section{ACKNOWLEDGEMENT}

The authors thank Ms. Mashaal Syed for her help with subject recruitment and data acquisition. This work was funded in part by a Drexel University College of Medicine and International Blue Cross Seed Grant \#1312002473 (Panait, PI) and a National Science Foundation (NSF) grant IIS: 1064871 (Shewokis, $\mathrm{PI})$. 


\section{INTRODUCTION}

Dramatic changes in health care and surgical training require novel, evidence-based methods to teach and assess procedural skills in and outside the operating room. The American College of Surgeons (ACS), the Association of Program Directors in Surgery (APDS) and Association of Surgical Education (ASE) have jointly developed skills curricula for medical students and surgical residents. ${ }^{1-3}$ However, the optimal method of skill acquisition using these national curricula remains to be defined. Recently the ASE simulation committee identified "defining the best methods/metrics to assess technical and nontechnical skills" as a top research priority. ${ }^{4}$ The committee suggested that technical skills can be measured with simple metrics such as time and dexterity, through the use of error-based checklists and global rating scales as well as with higher cognitive function tests such as functional brain imaging.

Functional near infrared spectroscopy (fNIR) is a noninvasive, optical brain imaging tool that monitors changes in total hemoglobin within the prefrontal cortex (PFC) in response to cognitive, motor and sensory activation (Figure 1). ${ }^{5-13}$ The PFC is responsible for motor planning, organization and regulation. It controls cognitive function and action, and works with other cortical circuits with executive functions including attention and working memory. ${ }^{14}$ Current fNIR systems are the size of a smartphone, run on battery power and wirelessly transfer immediate data, enabling assessment of brain activation in everyday settings and assessment of training. ${ }^{15}$

An important concept related to practice schedules and motor skill acquisition is contextual interference. Contextual interference is a learning phenomenon impacting how practice is organized when acquiring multiple similar tasks. Low contextual interference (blocked practice) is created when the tasks are presented in a sequential order while high contextual interference (random practice) occurs when the tasks are presented in a nonsequential order. ${ }^{16-19}$ Laparoscopic curricula utilize blocked ordered practice where learners perform the same task for a fixed number of times, or until a 
predefined proficiency level is achieved, before moving on to the next task. ${ }^{20}$ The assumptions for contextual interference studies are: 1) the acquisition of multiple, similar tasks/skills; 2) an equal numbers of trials per task are practiced, and 3) the only change in the design is how practice is scheduled or ordered. ${ }^{18,19}$ Based on the application of the assumptions, the following predictions are made: 1) during acquisition, learners who acquired the tasks in a blocked order would perform better than those acquiring the tasks in a RA order; 2) At the end of acquisition, blocked and random practice orders would perform similarly; and 3) during retention and transfer tests, learners who acquired the tasks in a random practice order would perform better than those learners who practiced the tasks in a BL order. ${ }^{18,19}$

Within surgical simulation training and testing, typically two aspects of motor learning practice parameters are combined, part and whole practice with contextual interference. ${ }^{4,17}$ The combination of the two practice parameters may be due to the complexity of surgical skills which necessitates the breakdown of tasks. ${ }^{16,17}$ Since 2005, several studies have implemented contextual interference paradigms for the assessment of surgical skill training. ${ }^{20-24}$ These studies assessed orthopedic boneplating, ${ }^{20,21}$ laparoscopic inguinal hernia repair, ${ }^{22}$ suturing skills, ${ }^{23}$ intracorporeal laparoscopic suturing and knot-tying ${ }^{5}$ and several Fundamentals of Laparoscopic Surgery (FLS) tasks. ${ }^{24}$ These studies had various amounts of practice ranging from 4-12 trials on the FLS task ${ }^{24}$ or 9 trials on a peg transfer task ${ }^{22}$ through 15 trials of suturing tasks, ${ }^{23} 60$ minutes of the bone-plating orthopedic surgical task ${ }^{21}$ and 8 hours of training on the intracorporeal laparoscopic suturing and knot-tying task. ${ }^{5}$ In the design of our study, we implemented varying degrees of task complexity (Figure 2) together with an increased number of trials during the training/acquisition phase and fNIR monitoring during performance of all trials. 
Using a contextual interference paradigm, we examined common behavioral performance metrics and brain-based biomarkers of prefrontal activation during novice acquisition, retention and transfer of selected simulated laparoscopic tasks performed on a virtual reality (VR) simulator. We hypothesized that during skill acquisition there will be higher global scores, lower total time and lower hemodynamics (i.e., cognitive effort) for the blocked relative to random order practice schedules. Also, during the acquisition phase we anticipate lower total time, higher global scores and attenuated cortical hemodynamics across the training sessions with random asymptoting to blocked at the end of acquisition. During retention and transfer, we hypothesize that random will have higher global scores, lower total time and lower mean change of total hemoglobin relative to blocked for each task. These hypotheses are based on the assumptions and predictions of contextual interference for behavioral measures $^{17-19}$ while the cortical hemodynamics are based on the role and influence of the prefrontal cortex during motor skill learning. ${ }^{29-33}$

\section{MATERIALS AND METHODS}

\section{Participants}

Fifteen third year medical students with no previous laparoscopic surgery volunteered for the study. Due to missing sessions and technical issues during fNIR recording, the data from five students was not analyzed. The remaining ten medical students were randomly assigned to either a blocked $(n=2 F ; n=3 M)$ or random $(n=2 F ; n=3 M)$ practice order. The medical students were medication-free, had normal or corrected-to-normal vision and provided written informed consent for participation in the study (Drexel University Institutional Review Board approved protocol \# 1312002473). 


\section{Experimental Protocol}

We used a repeated measures study design in which each student performed 36 trials each of three simulated laparoscopic tasks (LapSim ${ }^{\circledR}$ VR simulator) across three days, in either a blocked or random order, for the acquisition phase. Approximately 72 hours following skill acquisition, 6 retention ( 2 each of the acquisition tasks) and 6 transfer trials ( 3 trials of two new transfer task). fNIR monitoring of PFC activity occurred during all phases for the 16 optode sites (Figure 1).

\section{Laparoscopic Simulation Tasks}

During the acquisition phase of the study (Day 1 to Day 3), students practiced three skills (Camera Navigation, Lifting and Grasping and Fine Dissection) on a LapSim ${ }^{\circledR}$ VR simulator. These tasks correspond with basic laparoscopic skills on a task difficulty schematic of selected LapSim ${ }^{\circledR}$ and FLS tasks (Figure 2). Camera navigation reflected the skill of pointing a camera to a target at a correct angle and holding it steady (low on task difficulty); Lifting and grasping mimicked grasping a structure with an instrument, lifting and orienting it in a direction as instructed (slightly increased task difficulty). Fine dissection involved grasping a blood vessel with the non-dominant hand and bluntly dissecting the surrounding tissue with the dominant hand (much higher task difficulty). Two additional tasks were used to assess generalization in the skill transfer phase of the study. Simulated coordination involved pointing a camera at a target with non-dominant hand and this task was used to test for near transfer or interpolation. Simulated Laparoscopic Cholecystectomy was used to assess far transfer or extrapolation.

\section{Signal Processing}

The anterior prefrontal cortex of each participant was monitored using the fNIR 1100 system (fNIR Devices LLC; Potomac, MD; http://www.fnirdevices.com). The fNIR system is composed of three 
modules: a flexible headpiece sensor pad (Figure 1), which holds the light sources and detectors in a fixed integrated pad that facilitates placement; a control box for hardware management; and a computer for data acquisition. ${ }^{25}$

The positioning of the light sources and detectors on the sensor pad, anatomical landmarks for sensor placement consistency, data acquisition and visualization specifics and data processing details have been described. ${ }^{5,11,25}$ fNIR data epochs for each surgery simulation trial were extracted using time synchronization markers received through a serial port during the experiment. Hemodynamic changes for each of the 16 optodes during each trial were calculated separately using the Modified Beer Lambert Law. These data times were baseline corrected with respect to the start of the trial. To enhance spatial specificity, the final output of each optode was reported as the mean total hemoglobin $(\Delta \mathrm{HbT} \mu \mathrm{m}=$ deoxygenated hemoglobin + oxygenated hemoglobin). ${ }^{15,26}$

\section{Statistical Analyses}

Dependent measures included relative changes in mean $\Delta \mathrm{HbT}$ for four regions of interest within the anterior PFC (Right dorsolateral $=$ RDLPFC, Right medial $=$ RmidPFC, Left medial $=$ LmidPFC and Left dorsolateral $=$ LDLPFC), total time and global score performance metrics. We assessed if the acquisition data met the assumptions of normality, homogeneity of variance and sphericity for all dependent measures. For task acquisition, all dependent measures, $2 \times 2$ mixed-model ANOVAs with Practice Schedule (blocked versus random) as a between factor and Trial Block $(1,2,3)$ as a repeated measure were calculated separately. Post-hoc comparisons of significant interactions were computed using Tukey multiple comparison tests. Participants were considered a random-effect factor in this repeated measures design, whereas Practice Schedule and Trial Block factors were considered fixed-effects. Each Trial Block was comprised of 12 trials. To test a fixed-effect with one random effect in the model, the appropriate denominator term for the F-statistic was determined by limiting the error term for the interaction of the fixed and random factors to zero. ${ }^{27}$ The significance criterion for the tests was set at 
$\alpha=0.05$. In addition to the primary acquisition analyses, a separate set of analyses was conducted on the blocked data for each task. Average total hemoglobin change between blocked and random groups was compared using two-tailed independent t-tests for each simulated laparoscopic task.

For retention and transfer, we assessed if the data met the assumptions of normality and homogeneity of variance for all dependent measures. Independent t-tests were used to determine if there were differences between blocked versus random practice orders for each task separately. If the data were non-normally distributed, we used a Mann-Whitney $U$ non-parametric test. Effect sizes (Cohen's d) were calculated and used to aid in interpretation of the data. As a general rule for effect size interpretation, we used $d=0.2,0.5$ and $0.8>$, reflect small, medium and large effects, respectively. The significance criterion for all tests was set at $\alpha=0.05$. Number Cruncher Statistical Software (NCSS; www.ncss.com) was used for all the analyses.

\section{RESULTS}

\section{Performance Metrics}

\section{$\underline{\text { Acquisition }}$}

Performance metrics descriptive statistics and $p$-values from all the effects in the mixed model ANOVAs are reported in Tables $1 a, 1 b$ and $1 c$ for the camera navigation, lifting and grasping and fine dissection simulated laparoscopic tasks. The lifting and grasping Global Score (\%) was significant with random practice $(\mathrm{M} \pm \mathrm{SD} ; 94.9 \pm 8.8)$ scoring higher and it was less variable than blocked practice $(91.3$ \pm 10.0). Overall both practice schedules exhibited high competence with scores over $10 \%$ greater than minimal competency of $80 \%$. Blocked practice reached higher global scores faster than random practice for the camera navigation task (Table 1a and Figure 3a). There were significant differences for all tasks representing improvements in performance across time (Tables $1 \mathrm{a}, 1 \mathrm{~b}$ and $1 \mathrm{c}$, respectively).

\section{$\underline{\text { Retention and Transfer }}$}


For the simulated laparoscopic tasks, the t-tests between random and blocked practice groups for the global score and total time performance metrics along with descriptive statistics, $p$-values and effect sizes are reported in Table 2.

Analyzing total time, the random practice group consistently performed faster than blocked practice across camera navigation, lifting and grasping and the coordination tasks with medium to large effects $(d=0.59-1.18)$. During retention, for the camera navigation and lifting and grasping tasks, random practice performed the tasks faster and with less variability than blocked practice. In addition, the fine dissection simulated laparoscopic task showed a floor effect (blocked $=29.5 \pm 1.7$ versus random $=28.2 \pm 2.6 \mathrm{sec} ; p=0.210)$. For transfer, the coordination task showed trends $(p=0.055$ and $p=$ 0.098) for total time and global scores, respectively. For the cholescystectomy task there was a trend for global score $(p=0.068)$. In both transfer tasks, random practice had global scores greater than $80 \%$ proficiency, with lower variability relative to blocked practice (Table 2).

\section{$\Delta \mathrm{HbT}$ (Change Total Hemoglobin $\mu \mathrm{m})$}

The optodes of the anterior PFC were averaged across the LDLPFC (average of optodes \#1 - \#4), the LmidPFC (average of optodes \#5 - \#8), RmidPFC (average of optodes \#9 - \#12) and RDLPFC (average of optodes \#13 - \#16) regions (Figure 1) with mean values ( $\mu$ molar) assessed for all acquisition main effects and interactions for camera navigation, lifting and grasping and fine dissection simulated laparoscopic tasks (Tables $1 \mathrm{a}, 1 \mathrm{~b}$ and $1 \mathrm{c}$, respectively). For the retention and transfer simulated laparoscopic tasks, the results of the t-tests between random practice and blocked practice groups for $\Delta$ HbT along with descriptive statistics, p-values and effect sizes are reported in Table 3.

\section{$\underline{\text { Acquisition }}$}

For the simulated laparoscopic camera navigation task, there were significant interactions and main effects of Trial Block for all regions of interest (i.e., LDLPFC, LmidPFC, RmidPFC and RDLPFC for the mean $\Delta \mathrm{HbT}$ between the random practice and blocked practice groups (Table 1a). Mean $\Delta \mathrm{HbT}$ was 
higher for random practice versus blocked practice across the trial blocks (Figure 3a). Figure 4 depicts three topographical PFC maps. The left topographical map illustrates the contrast t-tests for mean $\Delta \mathrm{HbT}$ between blocked practice and random practice calculated for each of the 16 optodes. Teal through dark blue colors on the topographical map indicate random practice having increased effort relative to blocked practice, while orange and red colors indicate blocked practice having exerted more effort than random practice. For camera navigation, the RDLPFC and portions of the RmidPFC and LmidPFC show these random-blocked practice differences. The regions in which blocked practice exerted more effort than random practice are opposite of our predicted differences and they are seen in portions of the LmidPFC and RmidPFC.

The mean $\Delta \mathrm{HbT}$ differences for blocked versus random practice differences plotted across trial blocks for the lifting and grasping simulated surgical task are shown in Figure 3b (left). During acquisition of the lifting and grasping task, blocked practice exerted more effort across trial blocks than random practice. Table $1 \mathrm{~b}$, Figure $3 \mathrm{~b}$ and Figure 4 (center) illustrate that effort exerted by blocked practice was higher than random practice across trial blocks for lifting and grasping that is a somewhat more complex task than camera navigation (Figure 2).

For the simulated surgical fine dissection task, the results were more pronounced than seen in camera navigation and lifting and grasping tasks. Figure 4 (right) shows a topographical map of the contrast t-test of the mean $\Delta \mathrm{HbT}$ blocked-random differences calculated for each of the 16 optodes for the fine dissection task. As noted in Table 1c, Figures $3 c$ and Figure 4 (right), there were differences in the mean $\triangle \mathrm{HbT}$ for the LmidPFC. Similar to camera navigation, the effects for the LmidPFC in mean $\Delta \mathrm{HbT}$ were in the predicted direction. Thus, random practice used more cognitive effort than blocked practice to perform the fine dissection simulated surgical task.

Retention and Transfer 
For retention tasks, significant differences in the RDLPFC were detected for the mean $\triangle \mathrm{HbT}$ between the random and blocked practice groups for the camera navigation, lifting and grasping and fine dissection simulated surgical tasks. For the simulated surgical tasks, blocked practice used more mean $\mathrm{HbT}$ than random practice with very large effects $(\mathrm{d}>1.77$; Table 3 ). This translates to the random practice group using less cognitive effort in the RDLPFC for these tasks. Figure 5 depicts three topographical maps of the contrast t-tests for mean $\Delta \mathrm{HbT}$ between blocked and random practice calculated for each of the 16 optodes for camera navigation, lifting and grasping and fine dissection simulated VR tasks.

For the simulated surgical coordination transfer task, the results were comparable to those found in the retention tasks (Table 3). Figure 6 (left) shows a topographical map of the contrast t-test of the mean changes in the total hemoglobin calculated for each of the 16 optodes for the coordination transfer task. As noted in Table 3 and Figure $6 \mathrm{a}$, there were differences in the mean $\Delta \mathrm{HbT}$ for the RDLPFC. The cholescystectomy simulated transfer task also had significant differences between blocked and random practice groups in the RDLPFC (Table 3 and Figure 6 (right)). Similar to the retention tasks, the effects for the regions of interest in mean $\Delta \mathrm{HbT}$ were large to very large. Thus, random practice used less cognitive effort than blocked practice to perform the coordination and cholescystectomy simulated surgical tasks.

\section{DISCUSSION}

The purpose of this study was to examine contextual interference effects with simulated laparoscopic tasks during acquisition, retention and transfer phases using performance and cortical hemodynamic measures. Standard behavioral performance metrics (i.e. global scores and total time to task completion) were obtained from a LapSim ${ }^{\circledR}$ VR simulator, while cortical hemodynamic responses

were measured by a wearable headband optical brain imaging device that was worn by participants for 
all trials. We will discuss the effects of contextual interference during acquisition, retention and transfer phases with a focus on the influence of cortical hemodynamics and task difficulty on learning.

\section{Acquisition - Performance Metrics}

A primary feature of contextual interference is that a blocked practice order facilitates performance during acquisition relative to a random practice order. ${ }^{17-19}$ Our results indicate differential patterns for the performance metrics across the different practice schedules and tasks. Across the three acquisition tasks (camera navigation, lifting and grasping and fine dissection) there were no practice schedule differences for the total time performance metric. The composite global score, reflecting performance accuracy, speed, instrument trajectories and so forth, had a significant effect for blocked and random practice schedules for the lifting and grasping task (see Table 1b). The findings are in the opposite direction of the hypotheses. The lifting and grasping task incorporates several steps, involves some degree of bimanual coordination with manipulation of the laparoscopic instruments as well as an increased degree of task difficulty. A possible explanation for the reversal effect for random over blocked practice during acquisition may be explained by our design. Upon closer inspection of Table 1b, both blocked and random practice orders have levels of performance $>80 \%$ which is the minimum competency on the global scores. The blocked group practiced the same task for 36 trials in a single day, while the random group practiced 12 trials of each task each day. Perhaps there was a small amount of fatigue influencing the blocked group as evidenced by the slight drop in their scores of $95.7 \%$ to $92.2 \%$ from trial block 2 to trial block 3 . In addition, blocked practice showed an increase in variability from trial block 2 to trial block 3. The tasks and performance metrics findings are similar to those of Brydges et al ${ }^{20}$ with no acquisition blocked versus random practice differences for several of their tasks (reduction and application of a bone plate, bone-tapping and the insertion of screws). They found predicted acquisition $\mathrm{BL}$ advantage for the simpler skills of drilling and depth measurement. 
Although our findings are mixed relative to our hypotheses and the predictions of contextual interference during acquisition, the results help us to understand more about the role of practice organization, task difficulty and cognitive effort when novices acquire surgical skills. Contextual interference, or practice organization, based on our findings show that during the acquisition of simulated laparoscopic skills of varying levels of difficulty, random practice trended in high levels of competency (global score) across the trial blocks relative to blocked practice. Both practice orders were above the $80 \%$ minimum competency level. Other contextual interference applications of surgical skills like FLS skills, ${ }^{24}$ peg transfer task, ${ }^{22}$ and suturing tasks, ${ }^{23}$ had fewer trials or time to complete the tasks across the acquisition phase. Our findings during task acquisition for total time and global score on the high fidelity laparoscopic simulator are similar to the findings of Dumbrowski and colleagues ${ }^{20,21}$ with a low fidelity simulated bone-plating surgical task. The amount of practice time in these studies was similar to our acquisition phase of $60-90$ minutes of training. Given the complexity of laparoscopic surgical skills, it is essential to have a necessary amount of practice time to allow the learner to sufficiently perform the task. Previous work by Crewther et $a l^{6}$ had novices practice set-sequences of intracorporeal suturing and knot tying for a baseline of 120 minutes. Taken together, when training novice surgeons a baseline of training of 90-120 minutes is recommended.

Task difficulty is another primary factor that influenced our results. Figure 2 depicts a schematic of estimated task difficulty of several simulated tasks on the LapSim ${ }^{\circledR}$ VR simulator and the FLS tasks. These simulated laparoscopic task classifications were verified by expert surgeons and based on the number of task components, estimated time to task completion, use of the task or components in an actual surgery and the possibility for error on completion of the task. The three surgical tasks in the study; camera navigation, grasping and lifting and fine dissection, were clearly in the lower half of the task difficulty continuum. These tasks are similar in difficulty to two of the FLS tasks, peg transfer and precision cutting. We may have found no acquisition differences between blocked and random practice 
groups with our tasks because of the fact that we had longer practice with the tasks than Rivard et al ${ }^{24}$ who practiced the FLS tasks with a small and varying number of trials or Hernandez-Irizarry et al ${ }^{22}$ with the peg transfer task as a preliminary task in mastery learning of a laparoscopic inguinal hernia surgical task. We also consider another aspect of practice conditions, part practice and whole practice of a number of skill components or a skill. Although relatively simple on the task scale (Figure 2), camera navigation and grasping lifting tasks involves multiple components to successfully complete each task. It may be that each task would benefit from additional breakdown of sub-skills, where practice could be organized so that the sub-skills were practiced in a progressive part practice manner and compare this practice order to whole practice to facilitate acquisition. ${ }^{20,21,28}$ As well, performance at the end of acquisition is not the best indicator of learning. Learning is best illustrated by retention and transfer tests. ${ }^{17,21,22,24,27}$ We present our retention and transfer results later in the discussion.

\section{Acquisition - Changes in Cortical Hemodynamics}

Including a neurophysiological assessment of cognitive effort was an important aspect of our study and our unique contribution to the literature as our work helps to illustrate some of the differences in prefrontal cortex activation during surgical skill performance using different practice schedules. Regardless of the practice schedule for skill acquisition, prefrontal cortex activation is necessary in the early stages of learning motor skills especially for complex, attention-demanding tasks like surgical skills. ${ }^{29-33,38}$ Other investigations of surgical techniques have used a single task, e.g., knot tying, ${ }^{8}$ endoscopic suturing and knot-tying, ${ }^{9}$ laparoscopic intracorporeal suturing and knot-tying in a set sequence $^{6}$ and a laparoscopic suturing drill, ${ }^{14}$ that was performed in either a constant or blocked practice order. Our findings clearly illustrate (Tables $1 \mathrm{a}, 1 \mathrm{~b}$ and $1 \mathrm{c}$; Figures $3 \mathrm{a}, \mathrm{b}$ and $\mathrm{c}$ ) how there is additional effort expended by random practice in the prefrontal cortical regions during the acquisition of simulated surgical tasks. This finding is expected as reported by neural correlates of motor skill practice order reviews by Lage et $a l^{32}$ and Wright et $a l^{38}$ using either functional magnetic resonance imaging, 
diffusion magnetic resonance imaging or transcranial magnet stimulation. Although there are few motor learning studies that have investigated neurobiological measures, the prefrontal regions of activation are an important starting point for understanding motor skill acquisition and the stages of learning. Lage ${ }^{32}$ reported that the RDLPFC showed increased activation for RA practice at the end of acquisition as well as demonstrating increased DLPFC activation across acquisition. Our results for the simulated laparoscopic surgical tasks in acquisition show mixed results to those reported by Lage. ${ }^{32}$ This finding may be a result of the fact that the tasks reported in Lage were very simple tasks like sequence keypresses and go- no-go key pressing tasks. Laparoscopic tasks are more complex and consequently additional practice may be needed to clearly determine the impact of practice schedule during learning. Perhaps a two or three-fold increase in the amount of practice of these simulated surgical skills is needed so that automaticity or close to automaticity may be attained and would be comparable to the training durations incorporated by Shetty and colleagues. ${ }^{10}$

We tested novices in our study and showed some different hemodynamic patterns during acquisition than others who have tested novices on simulated surgical tasks. ${ }^{8-10}$ Shetty et al ${ }^{10}$ had a group of novice surgeons complete eight hours of training with a simulated laparoscopic surgical suturing skill. At the end of training, the novices performed the suturing task at an expert level while their prefrontal oxygenated hemoglobin activation was 4 to 5 times greater than expert laparoscopists. Using the same task in a longitudinal investigation of skill acquisition and stress adaptations in laparoscopic surgery training and detraining, Crewthers et $a l^{6}$ also reported no attenuation of prefrontal cortex activity at the end of the acquisition training (300 minutes of training). Other applications of frontal lobe imaging of surgical performance involved endoscopic suturing and knot tying, where Ohuchida et $a l^{9}$ found novices and surgical trainees showed increased prefrontal activation relative to expert surgeons. In summary, incorporation of prefrontal cortex activation measures is an important 
component of training to assist program directors with assessment of the level of learning of their trainees and residents.

These differential findings provide an important foundation for additional studies to incorporate functional neuroimaging along with practice organization so that a better understanding of how to structure educational training of surgeons may occur.

\section{Retention and Transfer - Performance Metrics}

The counterintuitive outcome of contextual interference is manifested during the assessments of learning that occur during the retention and transfer phases. In our work, we had two groups acquire the simulated laparoscopic tasks in either a blocked or random practice order. After a 72-hour rest period, all medical students returned for an additional testing session that involved retention of the three acquired tasks and transfer of two novel tasks. The retention testing order was random while the transfer order was blocked. During retention, for two of the three acquired tasks, random practice resulted in lower performance times to complete the camera navigation and lifting and grasping simulated laparoscopic tasks as compared to the blocked practice group (Table 2). Our findings are supported by Dubrowski et $\left.a\right|^{21}$ who tested a bone-plating task and found that during retention their random and whole practice groups performed better than their blocked group during a retention test. The fine dissection task, which was more difficult than camera navigation and lifting and grasping, found no blocked versus random practice differences which was similar to Rivard et al ${ }^{24}$ findings with selected FLS tasks during immediate and delayed retention tests.

In addition, for the coordination simulated surgical transfer task, the same benefits of random practice training were found, lower total time and a global score above the minimum competency of $80 \%$ compared to BL training. Although significance was not attained in the coordination task for both variables (Table $2-p=0.055$ and $p=0.098$ for total time and global score, respectively), the scores were in the predicted direction. In this paper, we focused on only the medical students who completed 
all of the phases of the study. We reported a significant reduction $(p=0.023)$ for random practice relative to blocked practice in the coordination task with an increase in the sample size of one subject (N-11) who completed the tasks (Table 1 and Figure 6$)^{39}$. The cholescystectomy transfer task had no significant differences for both total time and global score. We attribute no differences to the increased task difficulty, novice experience level as well as the low sample size.

\section{Retention and Transfer - Changes in Cortical Hemodynamics}

Our results indicate differential patterns for performance metrics and optical imaging results for the distinctive practice orders and tasks. Although there were no retention practice schedule differences for several of the performance metrics measures, we did detect differences between the blocked and random practice schedules for the mean $\Delta \mathrm{HbT}$ measure of cognitive effort. For all retention and transfer simulated laparoscopic tasks, the RDLPFCs showed random practice had lower total hemoglobin values, which means that the learners performed the task with less cognitive load (lower effort) than those learners who acquired the tasks in a blocked practice order (Table 3 and Figures $5 \& 6)$. In their review, Lage and colleagues ${ }^{32}$ noted that the DLPFC is attenuated in the random practice group relative to the blocked practice group. Essentially, random practice during the skill acquisition training forced the learners to use more effort to acquire the tasks. This additional effort benefitted the random practice group during retention and transfer for the simulated laparoscopic tasks. Acquiring tasks (camera navigation, lifting and grasping, fine dissection) under a random practice order facilitated the same cognitive and behavioral processes that were needed to perform the coordination task in transfer, which resulted in higher performance and lower cognitive effort relative to blocked practice. Although considered a basic laparoscopic skill, the fine dissection surgical skill was more difficult than the camera navigation and lifting and grasping simulated skills. Perhaps, one of the other basic laparoscopic surgical skills such as cutting, clip application or instrument navigation would have been more similar to the cognitive and psychomotor demands of the camera navigation and lifting 
and grasping tasks. Our findings support our hypothesis that random practice facilitates enhanced memory and generalization by better performance and lower neural demands (i.e., cognitive effort) during the retention and transfer of simulated surgical laparoscopic tasks.

Our results provide preliminary information about optical imaging measures of the DLPFCs and anterior PFC hemodynamic responses and its relationship to the acquisition, memory tests and generalizations of skill learning or transfer of simulated surgical laparoscopic tasks. These findings are comparable to those noted by Shewokis and colleagues ${ }^{25,40}$ using functional optical imaging and 3-D spatial navigation tasks and 3-D UAV piloting tasks. ${ }^{5}$ In addition, using fMRI, Wymbs and Grafton ${ }^{41}$ reported that the left inferior frontal gyrus (optode \#2 - Figure 1 left) was differentially activated during late learning as a function of practice schedule for the sequence execution of a go/no-go task. Shewokis et $a l^{40}$ showed transfer results illustrating that there is a differential relative mean oxygenation of the left inferior frontal gyrus region (optode \#2 - Figure 1 left) for random compared to blocked practice orders for virtual spatial navigation tasks. These results help to extend our understanding of the contextual interference effect regarding the influences of the practice order and task type on neural function. ${ }^{11,25,32,35,37,38,40,41}$

fNIR, because it is non-invasive, portable, small and mobile, has the potential to be used in future learning/training environments to provide objective, task related brain-based measures for assessing cognitive effort and neural processing during the learning process. Our findings support Seymour and colleagues ${ }^{4,42}$ and set the stage for additional learning scenarios using applications of simulation and practice organization within surgical training and medical education. ${ }^{4,6,10,11,20-24,33-35,42}$

In conclusion, we demonstrate that novice learners practicing in a random practice order will learn multiple simulated laparoscopic tasks more efficiently and effectively than learners practicing in a blocked practice order. Our results indicate that optical imaging can be used to capture learning effects in a simulated laparoscopic environment. By incorporating optical imaging during practice and testing 
assessments, surgical skills curricula can be designed and personalized to the individual learner.

Furthermore, given fNIR's portability and safety, this optical imaging modality is well suited for

constructing learning assessments and protocols that meet the current demands of surgical training.

\section{REFERENCES}

1. American College of Surgeons. ACS/ASE Medical Student Simulation-Based Surgical Skills Curriculum. Available from: http://web2.facs.org/medicalstudents/landing.cfm.

2. American College of Surgeons. ACS/APDS/ASE Resident Prep Curriculum. Available from: https://www.facs.org/education/program/resident-prep.

3. American College of Surgeons. Surgery Resident Skills Curriculum. Available from: https://www.facs.org/education/program/resident-skills.

4. Johnston MJ, Paige JT, Aggarwal R, et al. An overview of research priorities in surgical simulation: what the literature shows has been achieved during the 21st century and what remains. Am J Surg 2016;211:214-25.

5. Ayaz $\mathrm{H}$, Shewokis PA, Bunce $\mathrm{S}$, et al. Optical brain monitoring for operator training and mental workload assessment. Neuroimage 2012;59:36-47.

6. Crewther BT, Shetty K, Jarchi D, et al. Skill acquisition and stress adaptations following laparoscopic surgery training and detraining in novice surgeons. Surgical Endoscopy 2016;30:2961-8.

7. Gentili RJ, Shewokis PA, Ayaz H, Contreras-Vidal JL. Functional near-infrared spectroscopy-based correlates of prefrontal cortical dynamics during a cognitive-motor executive adaptation task. Frontiers in Human Neuroscience 2013;7:277:1-13.

8. Leff DR, Orihuela-Espina F, Atallah L, et al. Functional prefrontal reorganization accompanies learning-associated refinements in surgery: a manifold embedding approach. Computer Aided Surgery 2008;13:325-39.

9. Ohuchida K, Kenmotsu H, Yamamoto A, et al. The frontal cortex is activated during learning of endoscopic procedures. Surgical Endoscopy 2009;23:2296-301.

10. Shetty K, Leff DR, Orihuela-Espina F, et al. Persistent prefrontal engagement despite improvements in laparoscopic technical skill. JAMA Surgery 2016;151:682-4.

11. Shewokis PA, Ayaz H, Panait L, et al. Brain-in-the-Loop Learning Using fNIR and Simulated Virtual Reality Surgical Tasks: Hemodynamic and Behavioral Effects. In: Schmorrow DD, Fidopiastis MC, eds. Foundations of Augmented Cognition: 9th International Conference, AC 2015, Held as Part of $\mathrm{HCl}$ International 2015, Los Angeles, CA, USA, August 2-7, 2015, Proceedings. Cham: Springer International Publishing; 2015. p. 324-35.

12. Strangman G, Boas DA, Sutton JP. Non-invasive neuroimaging using near-infrared light. Biological Psychiatry 2002;52:679-93.

13. Villringer A, Chance B. Non-invasive optical spectroscopy and imaging of human brain function. Trends in Neurosciences 1997;20:435-42.

14. Procyk E, Goldman-Rakic PS. Modulation of dorsolateral prefrontal delay activity during selforganized behavior. J Neurosci 2006;26:11313-23.

15. Ayaz H, Onaral B, Izzetoglu K, et al. Continuous monitoring of brain dynamics with functional near infrared spectroscopy as a tool for neuroergonomic research: empirical examples and a technological development. Frontiers in Human Neuroscience 2013;7:871:1-13.

16. Palter VN, Orzech N, Reznick RK, Grantcharov TP. Validation of a structured training and assessment curriculum for technical skill acquisition in minimally invasive surgery: a randomized controlled trial. Annals of Surgery 2013;257:224-30. 
17. Shewokis PA. Memory consolidation and contextual interference effects with computer games. Perceptual and Motor Skills 2003;97:581-9.

18. Shea JB, Morgan RI. Contextual interference effects on the acquisition, retention and tansfer of a motor skill. Journal of Experimental Psychology: Human Learning and Memory 1979;5:179-187.

19. Magill RA, Hall KG. A review of the contextual interference effect in motor skill acquisition. Human Movement Science 1990;9:241-289.

20. Brydges R, Carnahan H, Backstein D, Dubrowski A. Application of motor learning principles to complex surgical tasks: searching for the optimal practice schedule. Journal of Motor Behavior 2007;39:40-8.

21. Dubrowski A, Backstein $D$, Abughaduma $R$, et al. The influence of practice schedules in the learning of a complex bone-plating surgical task. Am J Surg 2005;190:359-63.

22. Hernandez-Irizarry R, Zendejas B, Ali SM, Farley DR. Optimizing training cost-effectiveness of simulation-based laparoscopic inguinal hernia repairs. Am J Surg 2016;211:326-35.

23. Safir O, Williams CK, Dubrowski A, et al. Self-directed practice schedule enhances learning of suturing skills. Can J Surg 2013;56:E142-7.

24. Rivard JD, Vergis AS, Unger BJ, et al. The effect of blocked versus random task practice schedules on the acquisition and retention of surgical skills. Am J Surg 2015;209:93-100.

25. Ayaz H, Shewokis PA, Curtin A, et al. Using MazeSuite and functional near infrared spectroscopy to study learning in spatial navigation. Journal of Visualized Experiments:JOVE 2011 (56).

26. Abdelnour AF, Huppert T. Real-time imaging of human brain function by near-infrared spectroscopy using an adaptive general linear model. Neuroimage 2009;46(1):133-43.

27. Maxwell SE, Delaney HD. Designing Experiments and Analyzing Data: A Model Comparison Perspective. $2^{\text {nd }}$ edition. Mahwah, NJ: Lawrence Earlbaum. 2004.

28. Schaverien MV. Development of expertise in surgical training. Journal of Surgical Education 2010;67(1):37-43.

29. Miller LB. The human frontal lobes: An introduction. In The Human Frontal Lobes; Functions and Disorders. NY. Guiliford Press. 2007.

30. Dayan E, Cohen LG. Neuroplasticity subserving motor skill learning. Neuron. 2011;72:443-454.

31. Debaere F, Wenderoth N, Sunaert S, Va Hecke P, Swinnen SP. Changes in brain activation during the acquiistion of a new bimanual coordination task. Neuropsychologia 2004;42:855-867.

32. Lage, GM, Ugrinowitsch H, Apolinario-Souza T, Vieira MM, Albuquerqe MR, Benda RN. Repetition and variation in motor practice: A review of neural correlates. Neuroscience and Biobehavioral Reviews. 2015;57:132-141.

33. Leff DR, Leong JJH, Aggarwal R, Yang G-Z, Darzi A Could variations in technical skills acquisition in surgery be explained by differences in cortical plasticity? Ann Surg 2008;247:540-543.

34. Gallagher AG, Jordan-Black JA, O'Sullivan GC. Prospective, randomized assessment of the acquisition, maintenance, and loss of laparoscopic skills. Ann Surg 2012;256:387-393.

35. Spruit EN, Band GPH, Hamming JF. Increasing efficiency of surgical training: Effects of spacing practice on skill acquisiiton and retention in laparoscopy training. Surg Endoscopy 2015;29:22352243.

36. Dawe SR, Windsor JA, Broeders JAJL, Cregan PC, Hewett, PJ, Maddern Gj. A systematic review of surgical skills transfer after simulation-based training. Ann Surg 2014;259:236-248.

37. Mussgen DM, Ullen F. Transfer in motor sequence learning: Efffects of practice schedule and dequence context. Frontiers in Human Neuroscience 2015;642:1-13.

38. Wright D, Verwey W, Buchanen J, Chen J, Rhee J, Immink M. Consolidating behavioral and neurophysiologic findings to explain the influence of contextual interference during motor sequence learning. Psychol Bull Rev 2016;23:1-21. 
39. Shewokis PA, Shariff FU, Ayaz H, Greenawald L, Syed M, Liu Y, Uribe JI, Panait L, Castellanos AE, Lind DS. Educational neuroscience with fNIR and simulation training of surgical tasks. J Am Coll Surg, 2015, e9

40. Shewokis pA, Ayaz H, Curtin A, Izzetoglu K, Onaral B. Brain in the loop learning using functional near infrared spectroscopy. In D. Schmorrow \& C. Fidopiastis (Eds). Foundations of Augmented Cognition 2013, Vol 8027, 381-389. Springer Berlin Heidelberg.

41. Wymbs NF, Grafton ST. Neural substrates of practice structure that support future off-line learning. J Neurophysiol. 2009;102:2462-2476.

42. Seymour NE, Gallagher AG, Roman $S A$ et al., Virtual reality training improves operating room performance: Results of a randomized, double-blinded study. Ann Surg 2002;236:458-463; discussion 463-464. 


\section{Legends to the figure}

Figure 1: Functional Near Infrared Spectroscopy sensor overlayed on a brain image with the sensor configuration of light sources, detectors and optodes (left) and sensor (head band) covers forehead of surgeon (right).

Figure 2: Continuum of task difficulty for Simulated Surgical Laparoscopic Procedures, comparing FLS tasks with $\operatorname{LapSim}^{\circledast}$ used in this study.

Figure 3: Practice Schedule by Trial Block Interactions for the Mean Total Hemoglobin ( $\Delta \mu$ molar) for the Left Anterior Medial Prefrontal Cortex and Global Score (\%) for the Camera Navigation Simulated Laparoscopic Simulated Surgical Task.

Figure 4: Practice Schedule by Trial Block Interactions for the Mean Total Hemoglobin ( $\Delta \mu$ molar) for the Left Anterior Medial Prefrontal Cortex and Global Score (\%) for the Grasping and Lifting Simulated Laparoscopic Simulated Surgical Task.

Figure 5: Practice Schedule by Trial Block Interactions for the Mean Total Hemoglobin ( $\Delta \mu$ molar) for the Left Anterior Medial Prefrontal Cortex and Total Time s) for the Fine Dissection Simulated Laparoscopic Simulated Surgical Task.

Figure 6: Topographical maps of the Contrast T-test of mean changes in the Total Hemoglobin $(\Delta \mu$ molar) measured for calculated for each of the 16 optodes for Camera Navigation, Grasping \& Lifting(head band) covers forehead of participants (right) and sensor, and Fine Dissection Tasks. 
16 OPTODES

USING 10 LIGNT DETECTOAS
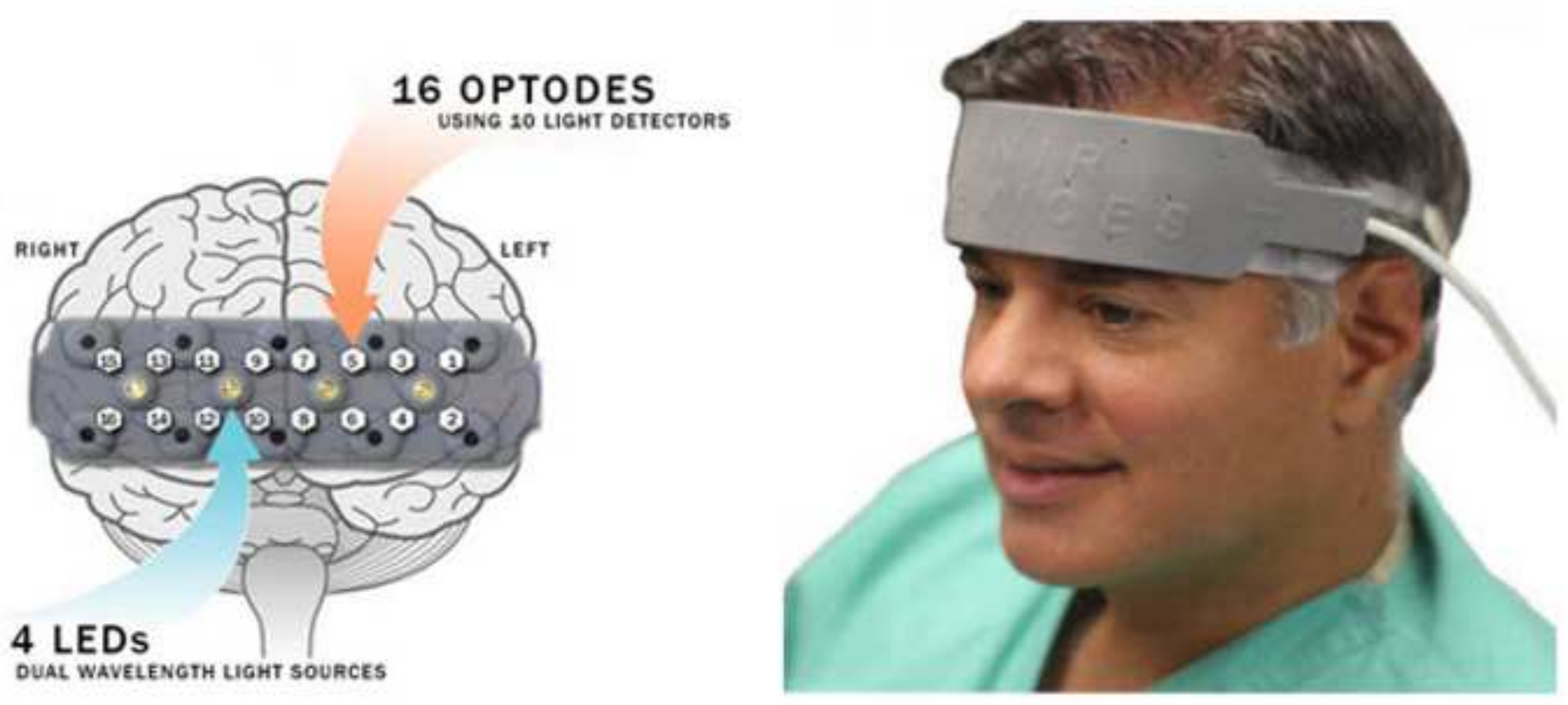

Figure

39

40

41

43

44

45

46

47

(

年

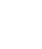




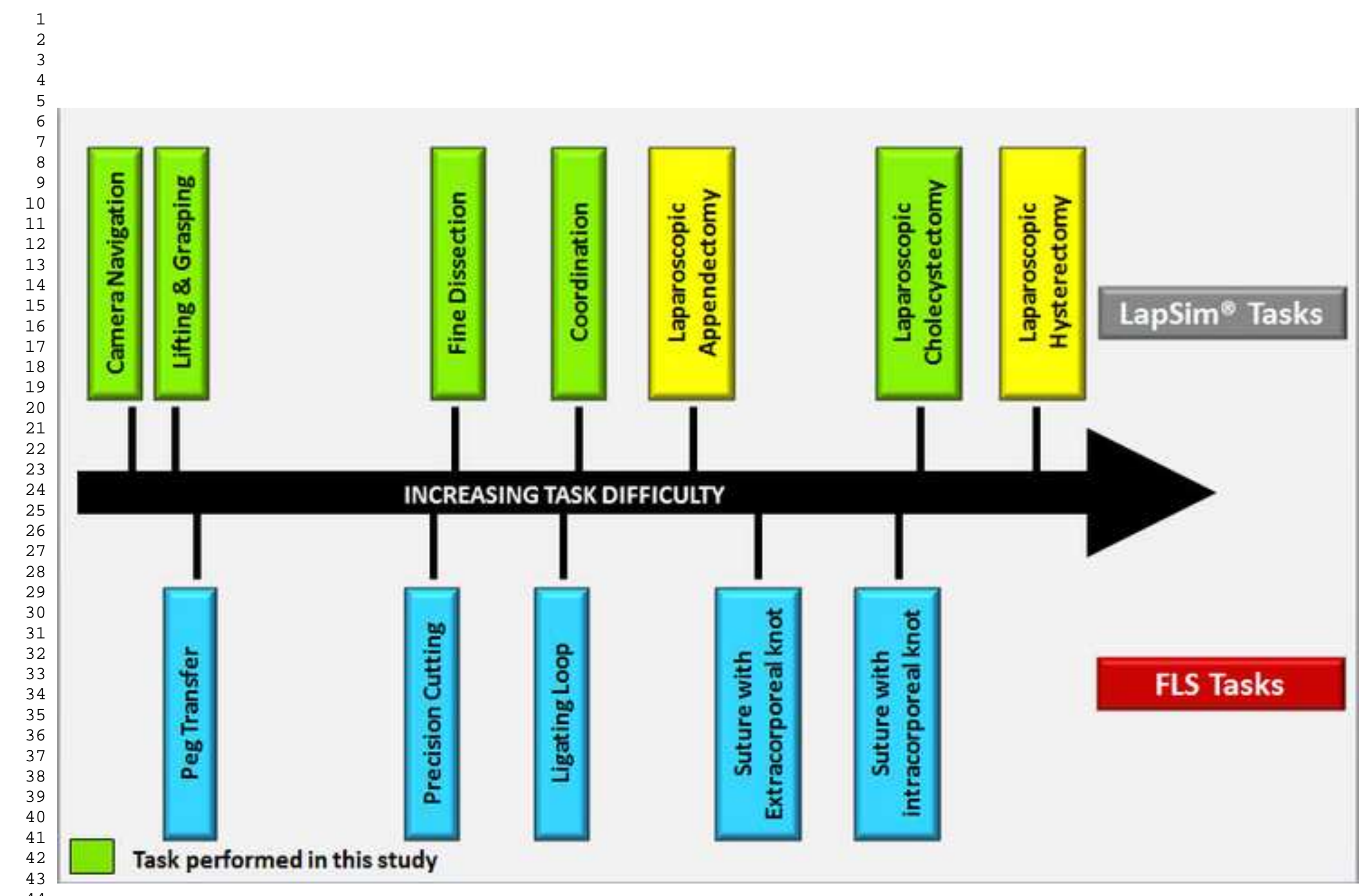

FLS Tasks 

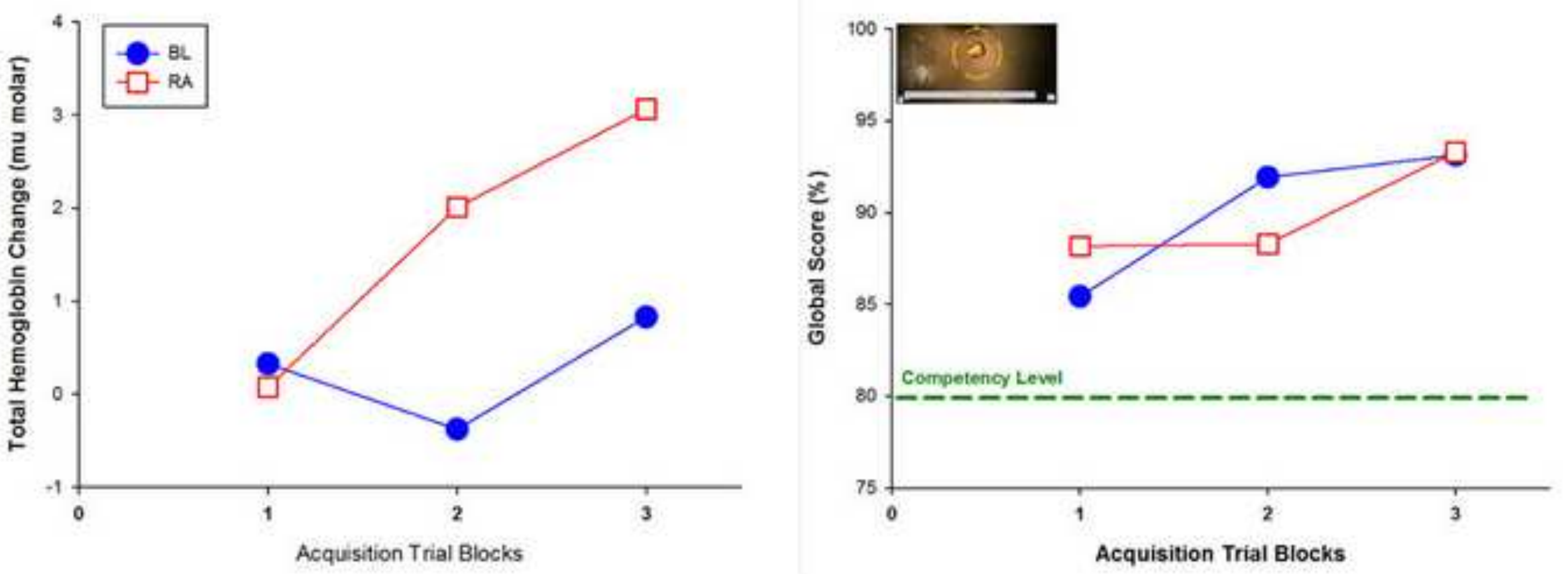

Figure 3

1
2

4

5
6

7

8

10

11

12
13

14

15
16

16
17

17
18
19

19
20

21

22

24

25

26

28

29

30

31

33

34

36

37

38

40
41

42

43

44

45

46

47 

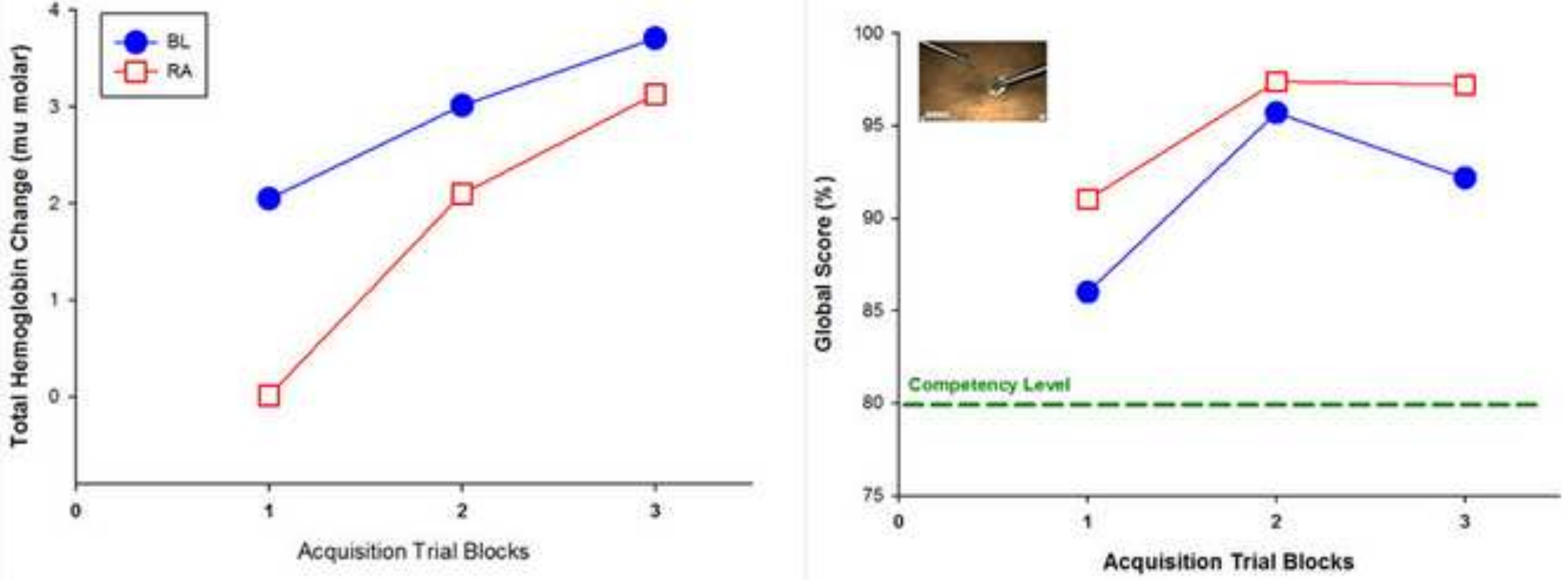

1
2

4

6

9

10

13

15
16

17

19

21

22

24

25

26

29

30

31

.

4

5
6
7
8
9



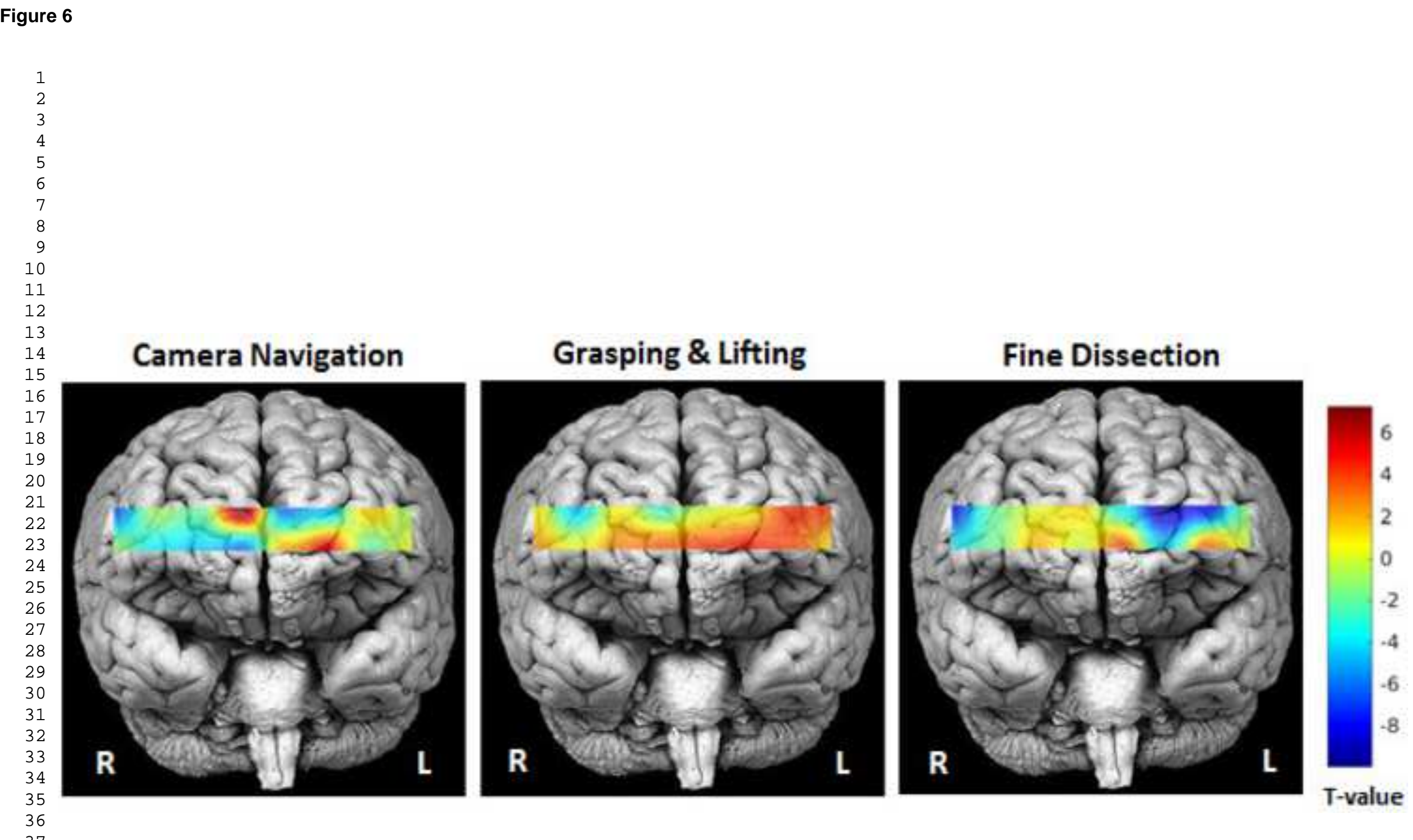

:

.

.

.

\section{Camera Navigation}

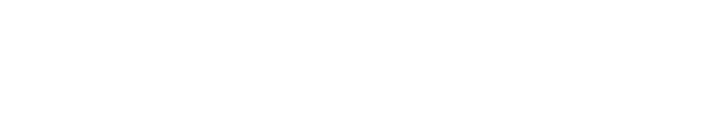

\section{Grasping \& Lifting}

T-value

Fine Dissection 
Table 1. Camera Navigation Performance Metrics and Prefrontal Cortex Hemodynamic Change Results

\begin{tabular}{|c|c|c|c|c|c|c|c|}
\hline $\begin{array}{l}\text { Dependent } \\
\text { Measures }\end{array}$ & $\begin{array}{c}\text { Practice } \\
\text { Schedule }\end{array}$ & $\begin{array}{c}\text { Trial Block } 1 \\
(M \pm S D)\end{array}$ & $\begin{array}{c}\text { Trial Block } 2 \\
\text { (M + SD) }\end{array}$ & $\begin{array}{c}\text { Trial Block } 3 \\
\text { (M + SD) }\end{array}$ & $\begin{array}{c}\text { Practice } \\
\text { Schedule X } \\
\text { Trial Block } \\
\text { p-value }\end{array}$ & $\begin{array}{c}\text { Trial Block } \\
\text { p-value }\end{array}$ & $\begin{array}{c}\text { Practice } \\
\text { Schedule } \\
\text { p-value }\end{array}$ \\
\hline \multirow{2}{*}{$\begin{array}{c}\text { Total Time } \\
\text { (s) }\end{array}$} & BL & $14.4 \pm 10.9$ & $11.9 \pm 9.3$ & $9.8 \pm 5.0$ & \multirow[t]{2}{*}{0.210} & \multirow[t]{2}{*}{$<0.001$} & \multirow[t]{2}{*}{0.856} \\
\hline & RA & $15.2+11.5$ & $9.6 \pm 5.0$ & $9.3 \pm 4.2$ & & & \\
\hline \multirow{2}{*}{$\begin{array}{c}\text { Global Score } \\
(\%)\end{array}$} & BL & $88.2 \pm 14.3$ & $88.3 \pm 14.9$ & $93.3 \pm 7.0$ & \multirow[t]{2}{*}{0.067} & \multirow[t]{2}{*}{$<0.001$} & \multirow[t]{2}{*}{0.954} \\
\hline & RA & $85.2 \pm 17.7$ & $91.8 \pm 8.7$ & $93.6 \pm 7.1$ & & & \\
\hline \multirow{2}{*}{$\begin{array}{c}\text { LDLPFC } \\
\Delta \mathrm{HbT}(\mu \mathrm{m})\end{array}$} & BI & $136+205$ & $216+223$ & $091+188$ & \multirow{2}{*}{$<0.001$} & \multirow{2}{*}{$<0.001$} & \multirow{2}{*}{0.642} \\
\hline & RA & $0.50+1.98$ & $2.20+2.32$ & $3.14+2.34$ & & & \\
\hline \multirow{2}{*}{$\begin{array}{c}\text { LmidPFC } \\
\Delta \mathrm{HbT}(\mu \mathrm{m})\end{array}$} & BL & $0.33 \pm 4.53$ & $-0.39+6.11$ & $0.83 \pm 3.20$ & \multirow[t]{2}{*}{$<0.001$} & \multirow[t]{2}{*}{$<0.001$} & \multirow[t]{2}{*}{$0.398^{\prime}$} \\
\hline & RA & $0.17 \pm 1.90$ & $2.36 \pm 2.18$ & $3.71 \pm 3.11$ & & & \\
\hline \multirow{2}{*}{$\begin{array}{c}\text { RmidPFC } \\
\Delta \mathrm{HbT}(\mu \mathrm{m})\end{array}$} & BL & $-0.82 \pm 4.36$ & $0.00 \pm 5.51$ & $0.02 \pm 1.71$ & \multirow[t]{2}{*}{0.008} & \multirow[t]{2}{*}{$<0.001$} & \multirow[t]{2}{*}{0.123} \\
\hline & RA & $0.37 \pm 2.61$ & $2.54 \pm 2.70$ & $3.86 \pm 3.23$ & & & \\
\hline \multirow{2}{*}{$\begin{array}{c}\text { RDLPFC } \\
\Delta \mathrm{HbT}(\mu \mathrm{m})\end{array}$} & $\mathrm{BL}$ & $0.63 \pm 2.79$ & $1.07 \pm 3.42$ & $-0.36 \pm 4.08$ & \multirow[t]{2}{*}{$<0.001$} & \multirow[t]{2}{*}{0.011} & \multirow[t]{2}{*}{0.367} \\
\hline & RA & $1.02+2.42$ & $2.22+2.62$ & $2.93+3.13$ & & & \\
\hline
\end{tabular}

Note: M - mean, SD - standard deviation; BL- Blocked, RA- Random; LDLPFC - left dorsolateral prefrontal cortex, LmidPFC - left anterior medial prefrontal cortex, RmidPFC - right anterior medial prefrontal cortex, RDLPFC - right dorsolateral prefrontal cortex; $\Delta \mathrm{HbT}$ ( $\mu \mathrm{m}$ ) - change in total hemoglobin (micromolar). For the Practice Schedule X Trial Block and Trial Block F test degrees of freedom were F(2, 277) while Practice Schedule was $[F(1,277)]$. 
Table 2. Lifting and Grasping Performance Metrics and Prefrontal Cortex Hemodynamic Change Results

\begin{tabular}{|c|c|c|c|c|c|c|c|}
\hline $\begin{array}{l}\text { Dependent } \\
\text { Measures }\end{array}$ & $\begin{array}{c}\text { Practice } \\
\text { Schedule }\end{array}$ & $\begin{array}{c}\text { Trial Block } 1 \\
(M \pm S D)\end{array}$ & $\begin{array}{c}\text { Trial Block } 2 \\
\text { (M + SD) }\end{array}$ & $\begin{array}{c}\text { Trial Block } 3 \\
\text { (M + SD) }\end{array}$ & $\begin{array}{c}\text { Practice } \\
\text { Schedule X } \\
\text { Trial Block } \\
\text { p-value }\end{array}$ & $\begin{array}{c}\text { Trial Block } \\
\text { p-value }\end{array}$ & $\begin{array}{c}\text { Practice } \\
\text { Schedule } \\
\text { p-value }\end{array}$ \\
\hline \multirow{2}{*}{$\begin{array}{l}\text { Total Time } \\
\text { (s) }\end{array}$} & BL & $38.9 \pm 17.2$ & $23.8 \pm 8.2$ & $24.7 \pm 9.5$ & \multirow[t]{2}{*}{0.181} & \multirow[t]{2}{*}{$<0.001$} & \multirow[t]{2}{*}{0.337} \\
\hline & RA & $33.1 \pm 12.1$ & $22.3 \pm 6.9$ & $20.0 \pm 6.9$ & & & \\
\hline \multirow{2}{*}{$\begin{array}{c}\text { Global Score } \\
(\%)\end{array}$} & BL & $86.0 \pm 13.5$ & $95.7 \pm 5.1$ & $92.2 \pm 10.0$ & \multirow[t]{2}{*}{0.381} & \multirow[t]{2}{*}{$<0.001$} & \multirow[t]{2}{*}{0.046} \\
\hline & RA & $90.3 \pm 12.7$ & $97.4 \pm 4.7$ & $97.0 \pm 4.7$ & & & \\
\hline \multirow{2}{*}{$\begin{array}{c}\text { LDLPFC } \\
\Delta \mathrm{HbT}(\mu \mathrm{m})\end{array}$} & BI & $166+181$ & $346+281$ & $287+200$ & \multirow{2}{*}{0.610} & \multirow{2}{*}{$<0.001$} & \multirow{2}{*}{0.432} \\
\hline & RA & $0.59+1.85$ & $2.14+2.24$ & $2.94+2.32$ & & & \\
\hline \multirow{2}{*}{$\begin{array}{c}\text { LmidPFC } \\
\Delta \mathrm{HbT}(\mu \mathrm{m})\end{array}$} & BL & $2.05 \pm 1.89$ & $3.15+1.79$ & $3.71 \pm 2.45$ & \multirow[t]{2}{*}{$<0.001$} & \multirow[t]{2}{*}{$<0.001$} & \multirow[t]{2}{*}{0.394} \\
\hline & RA & $0.17 \pm 2.26$ & $2.47 \pm 2.23$ & $3.76 \pm 2.98$ & & & \\
\hline \multirow{2}{*}{$\begin{array}{c}\text { RmidPFC } \\
\Delta \mathrm{HbT}(\mu \mathrm{m})\end{array}$} & BL & $1.59 \pm 1.68$ & $3.44 \pm 2.92$ & $3.97 \pm 3.01$ & \multirow[t]{2}{*}{0.218} & \multirow[t]{2}{*}{$<0.001$} & \multirow[t]{2}{*}{0.623} \\
\hline & RA & $0.44 \pm 2.63$ & $2.71 \pm 2.74$ & $3.73 \pm 3.19$ & & & \\
\hline \multirow{2}{*}{$\begin{array}{c}\text { RDLPFC } \\
\Delta \mathrm{HbT}(\mu \mathrm{m})\end{array}$} & $\mathrm{BL}$ & $1.33 \pm 3.05$ & $3.04 \pm 4.44$ & $3.07 \pm 4.18$ & \multirow[t]{2}{*}{0.493} & \multirow[t]{2}{*}{$<0.001$} & \multirow[t]{2}{*}{0.924} \\
\hline & RA & $1.44 \pm 2.46$ & $2.55 \pm 2.77$ & $2.86 \pm 2.78$ & & & \\
\hline
\end{tabular}

Note: M - mean, SD - standard deviation; BL- Blocked, RA- Random; LDLPFC - left dorsolateral prefrontal cortex, LmidPFC - left anterior medial prefrontal cortex, RmidPFC - right anterior medial prefrontal cortex, RDLPFC - right dorsolateral prefrontal cortex; $\Delta \mathrm{HbT}$ ( $\mu \mathrm{m})$ - change in total hemoglobin (micromolar). For the Practice Schedule X Trial Block and Trial Block F test degrees of freedom were F $(2,277)$ while Practice Schedule was $[F(1,277)]$. 
Table 3. Fine Dissection Performance Metrics and Prefrontal Cortex Hemodynamic Change Results

\begin{tabular}{|c|c|c|c|c|c|c|c|}
\hline $\begin{array}{l}\text { Dependent } \\
\text { Measures }\end{array}$ & $\begin{array}{l}\text { Practice } \\
\text { Schedule }\end{array}$ & $\begin{array}{c}\text { Trial Block } 1 \\
(M \pm S D)\end{array}$ & $\begin{array}{c}\text { Trial Block } 2 \\
(M+S D)\end{array}$ & $\begin{array}{c}\text { Trial Block } 3 \\
\text { (M + SD) }\end{array}$ & $\begin{array}{c}\text { Practice } \\
\text { Schedule } X \\
\text { Trial Block } \\
\text { p-value }\end{array}$ & $\begin{array}{c}\text { Trial Block } \\
\text { p-value }\end{array}$ & $\begin{array}{c}\text { Practice } \\
\text { Schedule } \\
\text { p-value }\end{array}$ \\
\hline \multirow{2}{*}{$\begin{array}{c}\text { Total Time } \\
\text { (s) }\end{array}$} & BL & $29.8 \pm 0.6$ & $29.2 \pm 2.2$ & $29.4 \pm 1.5$ & \multirow[t]{2}{*}{0.702} & \multirow[t]{2}{*}{0.014} & \multirow[t]{2}{*}{0.293} \\
\hline & RA & $30.0 \pm 0.3$ & $29.9 \pm 0.6$ & $29.4 \pm 1.6$ & & & \\
\hline \multirow{2}{*}{$\begin{array}{c}\text { Global Score } \\
(\%)\end{array}$} & BL & $96.2 \pm 13.1$ & $98.0+3.3$ & $97.4 \pm 4.2$ & \multirow[t]{2}{*}{0.225} & \multirow[t]{2}{*}{0.001} & \multirow[t]{2}{*}{0.876} \\
\hline & RA & $94.2 \pm 8.6$ & $98.6 \pm 3.2$ & $98.0 \pm 4.1$ & & & \\
\hline \multirow{2}{*}{$\begin{array}{c}\text { LDLPFC } \\
\Delta \mathrm{HbT}(\mu \mathrm{m})\end{array}$} & BL & $0.82 \pm 1.74$ & $1.64 \pm 1.89$ & $2.46 \pm 2.69$ & \multirow[t]{2}{*}{0.098} & \multirow[t]{2}{*}{$<0.001$} & \multirow[t]{2}{*}{0.856} \\
\hline & RA & $0.50 \pm 1.96$ & $2.10 \pm 2.02$ & $2.90 \pm 2.32$ & & & \\
\hline \multirow{2}{*}{$\begin{array}{c}\text { LmidPFC } \\
\Delta \mathrm{HbT}(\mu \mathrm{m})\end{array}$} & BL & $-0.27 \pm 2.30$ & $0.86 \pm 2.61$ & $1.20 \pm 2.23$ & \multirow[t]{2}{*}{$<0.001$} & \multirow[t]{2}{*}{$<0.001$} & \multirow[t]{2}{*}{0.237} \\
\hline & RA & $0.29 \pm 2.41$ & $2.34 \pm 2.40$ & $3.90 \pm 3.25$ & & & \\
\hline \multirow{2}{*}{$\begin{array}{c}\text { RmidPFC } \\
\Delta \mathrm{HbT}(\mu \mathrm{m})\end{array}$} & BL & $0.69 \pm 1.89$ & $2.87 \pm 1.87$ & $4.53 \pm 2.47$ & \multirow[t]{2}{*}{0.780} & \multirow[t]{2}{*}{$<0.001$} & \multirow[t]{2}{*}{0.733} \\
\hline & RA & $0.40 \pm 2.76$ & $2.48 \pm 2.70$ & $3.87 \pm 3.67$ & & & \\
\hline \multirow{2}{*}{$\begin{array}{c}\text { RDLPFC } \\
\Delta \mathrm{HbT}(\mu \mathrm{m})\end{array}$} & BL & $-0.25 \pm 1.70$ & $0.88 \pm 2.11$ & $1.37 \pm 2.46$ & \multirow[t]{2}{*}{0.938} & \multirow[t]{2}{*}{$<0.001$} & \multirow[t]{2}{*}{0.169} \\
\hline & RA & $1.13 \pm 2.41$ & $2.40 \pm 2.69$ & $2.92 \pm 2.94$ & & & \\
\hline
\end{tabular}

Note: M - mean, SD - standard deviation; BL- Blocked, RA- Random; LDLPFC - left dorsolateral prefrontal cortex, LmidPFC - left anterior medial prefrontal cortex, RmidPFC - right anterior medial prefrontal cortex, RDLPFC - right dorsolateral prefrontal cortex; $\Delta \mathrm{HbT}$ ( $\mu \mathrm{m}$ ) - change in total hemoglobin (micromolar). For the Practice Schedule X Trial Block and Trial Block F test degrees of freedom were F(2, 277) while Practice

Schedule was $[F(1,277)]$. 